\title{
SELETIVIDADE DE PRODUTOS FITOSSANITÁRIOS SOBRE O ÁCARO PREDADOR Agistemus brasiliensis Matioli, Ueckermann \& Oliveira (ACARI: STIGMAEIDAE) ${ }^{1}$
}

\author{
MARCOS ZATTI DA SILVA², CARLOS AMADEU LEITE DE OLIVEIRA ${ }^{3}$, MÁRIO EIDI SATO ${ }^{4}$
}

RESUMO - Os ácaros predadores das famílias Phytoseiidae e Stigmaeidae constituem-se nos principais inimigos naturais de Brevipalpus phoenicis (Geijskes) em citros. Este ácaro-praga causa sérios prejuízos na produção, devido à transmissão do vírus da leprose dos citros (CiLV). Apesar do grande volume de informações sobre a sensibilidade de ácaros Phytoseiidae a agrotóxicos, praticamente não existem informações sobre o efeito desses compostos em ácaros Stigmaeidae no Brasil. Sendo assim, o trabalho teve por objetivo avaliar o efeito dos principais agrotóxicos utilizados em citros sobre o ácaro predador Agistemus brasiliensis Matioli, Ueckermann \& Oliveira (Acari: Stigmaeidae), em condições de laboratório. Arenas de folhas de citros da variedade Pera, contendo 25 fêmeas adultas de A. brasiliensis, foram pulverizadas em torre de Potter. Avaliaram-se as mortalidades dos ácaros 72 horas após a aplicação. O efeito dos produtos na reprodução do acarino e a viabilidade dos ovos também foram avaliados. Quanto à seletividade, conforme proposta da “Organização Internacional para o Controle Biológico” (IOBC), os produtos foram classificados como: classe 1 - inócuo $(\mathrm{E}<30 \%)$, acrinathrin, bifenthrin, carbosulfan, deltamethrin; 2 - levemente nocivo $(30 \%<\mathrm{E}<79 \%)$, acefato, thiametoxam; 3 - moderadamente nocivo (80\%<E<99\%), abamectin, chlorfenapyr, chlorpyrifos, dicofol, dimetoato, dinocap; 4 - nocivo (E $>99 \%)$, calda sulfocálcica, cyhexatin, flufenoxuron, hexythiazox, óxido de fenbutatin, propargite, pyridaben e spirodiclofen. Estudos conduzidos em condições de campo ainda são necessários para se compreender melhor o efeito desses agrotóxicos sobre o ácaro predador.

Termos para indexação: inimigos naturais, citros, manejo integrado de pragas, controle biológico.

\section{SELECTIVITY OFTHE PESTICIDES TO THE PREDACEOUS MITE Agistemus brasiliensis Matioli,, Ueckermann \& Oliveira (ACARI: STIGMAEIDAE)}

\begin{abstract}
The predaceous mites of the families Phytoseiidae and Stigmaeidae are the most important natural enemies of Brevipalpus phoenicis (Geijskes) on citrus. This mite causes serious damages to the yield due to the transmission of Citrus leprosis virus (CiLV). Despite the considerable amount of information on susceptibility of phytoseiids to pesticides, the effect of these compounds is not very known for stigmaeid mites in Brazil. This work was carried out to evaluate the effect of the main pesticides used in citrus orchards on the predaceous mite Agistemus brasiliensis Matioli, Ueckermann \& Oliveira (Acari: Stigmaeidae) in laboratory conditions. Citrus leaf ("Pera" cultivar) arenas with 25 adult females of $A$. brasiliensis were sprayed in a Potter tower. The mortality of mites was assessed 72 hours after treatment. The effect of pesticides on the reproduction of mite and egg viability was also evaluated. According to the proposal of the Working Group of "International Organization for Biological Control” (IOBC), the pesticides were classified as: class 1 - harmless (E<30\%), acrinathrin, bifenthrin, carbosulfan, deltamethrin; 2 - slightly harmful (30\%<E<79\%), acephate, thiamethoxam; 3 - moderately harmful (80\%<E<99\%), abamectin, chlorfenapyr, chlorpyrifos, dicofol, dimethoate, dinocap; 4 harmful (E>99\%), lime sulfur, cyhexatin, flufenoxuron, hexythiazox, fenbutatin oxide, propargite, pyridaben and spirodiclofen. Studies in the field conditions are still necessary for a better understanding of the effect of these chemicals on the predaceous mite.
\end{abstract}

Index Terms: Natural enemies, citrus, integrated pest management, biological control.

${ }^{1}$ (Trabalho 093-08). Recebido em: 11-04-2008. Aceito para publicação em: 05-02-2009.

${ }^{2}$ Pós-Graduando em Agronomia-Entomologia Agrícola, FCAV-UNESP. makdsil@ig.com.br. Bolsista Capes.

${ }^{3}$ Prof. Titular Departamento de Fitossanidade, amadeu@fcav.unesp.br. Faculdade de Ciências Agrárias e Veterinária-UNESP, Via de acesso Prof. Paulo Donato Castellane s/n, CEP: 14884-900. Jaboticabal-SP.

${ }^{4}$ Pesquisador Científico, Laboratório de Entomologia Econômica, CEIB. mesato@biologico.sp.gov.br. Instituto Biológico - APTA. Caixa Postal 70, CEP 13001-970, Campinas-SP. 


\section{INTRODUÇÃO}

O ácaro Brevipalpus phoenicis (Geijskes) é uma das principais pragas da citricultura brasileira, devido aos sérios prejuízos que causa na produção. Este ácaro é responsável pela transmissão do vírus da leprose dos citros (CiLV), que está associado a sintomas como lesões localizadas em frutos, ramos e folhas, queda prematura de frutos, desfolhamento e morte de ramos, que levam as plantas a um sério declínio (Rodrigues et al., 2003).

A utilização de agrotóxicos para o controle de ácaros fitófagos presentes na cultura dos citros tem causado uma série de problemas, como o desenvolvimento de resistência de ácaros-praga a acaricidas, a eliminação dos inimigos naturais presentes na cultura e o favorecimento da ressurgência de pragas (Van de Vrie et al., 1972; Omoto et al., 2000).

Os ácaros predadores, quando abundantes na cultura, podem manter a população de ácaros fitófagos em níveis que não causem prejuízos econômicos, por um longo período após o tratamento químico, exigindo assim menor número de aplicações, reduzindo a pressão de seleção e, consequentemente, retardando o desenvolvimento da resistência dos ácaros-praga (Sato, 2005).

Um efetivo controle biológico do ácaro $B$. phoenicis pode resultar em redução significativa no nível de incidência da doença, uma vez que o agente causal não é circulativo (Kitajima et al., 1971). A redução numérica da população do ácaro transmissor pode levar à diminuição nos danos causados à cultura por essa doença (Moraes \& Sá, 1995).

Como agentes de controle biológico, destacamse os ácaros das famílias Phytoseiidae e Stigmaeidae (Moraes, 2002; Matioli \& Oliveira, 2007), sendo os pertencentes à família Phytoseiidae os mais estudados e utilizados em programas de controle biológico (McMurtry et al., 1970; Moraes, 1991). Stigmaeidae é considerada a segunda família de maior importância no controle de ácaros fitófagos (Gerson et al., 2003), com representantes encontrados com frequência em citros e outras culturas agrícolas de relevância econômica (Santos \& Laing, 1985; Sepasgosarian, 1985; Woolhouse \& Harmsen, 1994; Mineiro et al., 2008). Algumas espécies são referidas como predadoras de cochonilhas na América do Norte, Europa e Egito (Zaher \& Elbadry, 1961; Rasmy, 1975; Krantz, 1978).

Estudos relacionados à dinâmica populacional de ácaros estigmeídeos na cultura de citros foram realizados nos municípios de Limeira, Araraquara, Jaboticabal, Itápolis, Bebedouro e Olímpia, Estado de São Paulo, durante dois anos, onde foram amostrados frutos, ramos e folhas. Verificou-se que a espécie de estigmeídeo mais frequente foi Agistemus brasiliensis Matioli, Ueckermann \& Oliveira (80\%), seguida de Agistemus floridanus Gonzalez (15\%) e Zetzellia malvinae Matioli, Ueckermann \& Oliveira (5\%). Os ácaros estigmeídeos foram encontrados em maior número nos frutos, seguido das folhas de citros (Matioli, 2002).

A potencialidade de predação de $A$. brasiliensis foi estudada para adultos do ácaro-daleprose. Os estudos indicaram que a densidade de 20 ácaros-da-leprose por arena proporcionou ao estigmeídeo o consumo de 7,6 B. phoenicis por dia. Em relação à postura diária, verificou-se que densidades acima de 40 ácaros-da-leprose proporcionaram uma média de 4,7 ovos/fêmea/dia (Matioli \& Oliveira, 2007).

Apesar do grande volume de informações sobre a sensibilidade de ácaros Phytoseiidae a agrotóxicos em citros (Yamamoto et al., 1992; Sato et al., 1994, 1996; Reis et al., 1998, 1999; Silva \& Oliveira, 2006, 2007), praticamente não existem informações sobre o efeito destes compostos em ácaros Stigmaeidae no Brasil. Assim sendo, o objetivo do presente trabalho foi avaliar a seletividade dos principais agrotóxicos, com atividade acaricida e/ou inseticida, utilizados em citros sobre A. brasiliensis.

\section{MATERIAL E MÉTODOS}

A pesquisa foi realizada no Laboratório de Entomologia Econômica, do Centro Experimental Central do Instituto Biológico, em Campinas-SP. Os ácaros estigmeídeos utilizados neste estudo foram coletados em agosto de 2005, de plantas de laranjeira (Citrus sinensis), das variedades Pera e Valência, em pomares localizados no Câmpus da FCAV/UNESP em Jaboticabal-SP.

A identificação da espécie foi realizada pelo Dr. André Luiz Matioli, do Instituto Biológico, Campinas-SP.

Criação de Agistemus brasiliensis - A criação de ácaros estigmeídeos foi realizada em sala climatizada a $25 \pm 2^{\circ} \mathrm{C}$, umidade relativa de $70 \pm 10 \%$ e fotofase de $12 \mathrm{~h}$. Foram transferidos ácaros para folhas de laranjeira da variedade Pera, colocadas sobre camada de algodão hidrófilo em placa de Petri (15 cm de diâmetro). Para evitar a fuga de ácaros, manteve-se a borda da folha coberta com algodão umedecido. Ninfas e adultos de B. phoenicis e pólen de mamona, Ricinus communis L., ou de taboa, Typha sp., foram colocados em abundância em cada arena para servir de alimento aos ácaros predadores, conforme descrito por Komatsu (1988). O pólen de 
taboa foi obtido de acordo com Moreira (1993), e assim como o de mamona, foi armazenado por até 30 dias sob refrigeração $\left(4^{\circ} \mathrm{C}\right)$.

Criação de Brevipalpus phoenicis - Foram coletados ácaros de plantas de citros, de pomares não pulverizados, para iniciar a criação-estoque. Foram transferidos 40 a 50 ácaros adultos com o auxílio de um pincel de pelo macio, para frutos de laranja da variedade Pera ou Valência. Os frutos eram previamente lavados com água e secos e, em seguida, parafinados, deixando-se uma arena de aproximadamente 3 a $5 \mathrm{~cm}^{2}$ circundada com cola adesiva $\left(\right.$ Tanglefoot $^{\circledR}$ ), para a contenção dos ácaros. A criação foi mantida em sala climatizada à temperatura de $25 \pm 2^{\circ} \mathrm{C}$, umidade relativa de $70 \pm$ $10 \%$ e fotofase de $12 \mathrm{~h}$. Os frutos foram substituídos a cada 30 a 40 dias, à medida que deterioravam.

Delineamento experimental - O bioensaio, conduzido em laboratório, foi delineado experimentalmente em parcelas inteiramente casualizadas, onde 20 tratamentos foram repetidos quatro vezes. Cada unidade experimental foi constituída de uma arena de folha de laranjeira contendo 25 fêmeas adultas de A. brasiliensis. As arenas de folhas de citros da variedade Pera foram confeccionadas utilizando-se de um disco foliar de laranjeira de $4 \mathrm{~cm}$ de diâmetro, que foi colocado sobre uma camada de algodão hidrófilo, em uma placa de Petri de 9 cm de diâmetro.

Os acaricidas testados (Tabela1) foram empregados nas concentrações recomendadas para o controle do ácaro da leprose B. phoenicis no Brasil, e os demais agrotóxicos foram utilizados nas suas concentrações recomendadas para outras pragas de importância econômica na citricultura (Agrofit, 2008).

Arenas de folhas de citros contendo as fêmeas de $A$. brasiliensis foram pulverizadas em torre de Potter (Burkard Scientific, Uxbridge, UK), calibrada a 68,9 kPa. Utilizou-se um volume de $2 \mathrm{~mL}$ de calda, em cada pulverização, obtendo-se uma deposição média de resíduo úmido de $1,6 \mathrm{mg} / \mathrm{cm}^{2}$ da arena. A pulverização foi realizada sobre as fêmeas de $A$. brasiliensis que foram mantidas na superfície tratada até o final das avaliações. Após a aplicação, as arenas foram mantidas em câmara climatizada a $25 \pm 2^{\circ} \mathrm{C}, 70 \pm 10 \%$ de UR e fotofase de 12 horas.

Critérios de avaliação - Para a determinação do efeito dos agrotóxicos sobre adultos de $A$. brasiliensis, considerou-se a mortalidade observada 72 horas após a aplicação. Quase toda a mortalidade causada pelos agrotóxicos testados ocorre nas primeiras 72 horas, inclusive para os produtos de ação mais lenta, como propargite.

As avaliações foram realizadas com auxílio de um microscópio estereoscópico, consideraram-se mortos os ácaros predadores que não conseguiam locomover-se por uma distância mínima equivalente ao comprimento do seu corpo, ao serem tocados levemente com pincel de pelos macios.

Os dados relativos à mortalidade de $A$. brasiliensis foram submetidos à análise de variância, pelo teste F, e as médias comparadas pelo teste Tukey, a $5 \%$ de probabilidade. Os percentuais de mortalidade foram calculados e corrigidos segundo a fórmula de Henderson \& Tilton (1955).

$O$ efeito na reprodução $\left(E_{r}\right)$ foi calculado dividindo-se a produção média de ovos no tratamento $\left(\mathrm{R}_{\text {trat. }}\right)$ pela produção média de ovos na testemunha $\left(\mathrm{R}_{\text {test. }}\right)$. A produção média de ovos por fêmea $(\mathrm{R})$ foi obtida através da relação: $\mathrm{R}$ = total de ovos viáveis/ número de fêmeas vivas no final do teste. Foram considerados válidos somente os testes em que a mortalidade na testemunha foi de, no máximo, 20\% (Bakker et al., 1992).

O efeito total ou adverso (E\%) do produto foi calculado levando-se em consideração a mortalidade das fêmeas no tratamento, corrigida em função da mortalidade na testemunha, e o efeito na reprodução, através da fórmula: $\mathrm{E} \%=100 \%-\left(100 \%-\mathrm{M}_{\mathrm{c}}\right) \mathrm{x} \mathrm{E}_{\mathrm{r}}$, onde $\mathrm{M}_{\mathrm{c}}=$ mortalidade corrigida (Henderson \& Tilton, 1955) e $E_{r}=$ efeito na reprodução (Hassan et al., 1985; Overmeer, 1988; Bakker et al., 1992).

A avaliação da viabilidade dos ovos postos nas arenas, entre a aplicação e 72 horas após o tratamento, estendeu-se por um período de 7 dias, tempo suficiente para a eclosão das larvas nas condições do bioensaio.

Os valores dos efeitos totais obtidos para cada produto foram classificados conforme critérios estabelecidos pela IOBC, específicos para organismos benéficos em testes de laboratório (Bakker et al., 1992; Hassan et al., 1994), sendo classe 1: $\mathrm{E}<30 \%$ (inócuo, não-nocivo); classe 2: 30\%< E $<79 \%$ (levemente nocivo); classe 3: 79\% $<\mathrm{E}<99 \%$ (moderadamente nocivo), e classe 4: $\mathrm{E}>99 \%$ (nocivo).

\section{RESULTADOS E DISCUSSÃO}

Os produtos calda sulfocálcica, cyhexatin, óxido de fenbutatin, propargite e pyridaben foram nocivos ao estigmeídeo $A$. brasiliensis, causando $100 \%$ de mortalidade dos ácaros (Tabela 2). Resultados semelhantes aos verificados no presente trabalho foram obtidos para o ácaro Agistemus industani Gonzales (Stigmaeidae) em citros, na Flórida/EUA, quando se pulverizaram os acaricidas: 
óxido de fenbutatin, propargite e pyridaben (Childers et al., 2001).

Trabalhos realizados no Brasil para avaliar a seletividade de agrotóxicos a ácaros predadores da família Phytoseiidae, presentes em citros, também indicaram alta toxicidade dos acaricidas cyhexatin, óxido de fenbutatin, propargite e pyridaben a algumas espécies de fitoseídeos (Yamamoto et al., 1992; Sato et al., 1995, 1996; Reis et al., 1999; Silva \& Oliveira, 2006, 2007).

De acordo com Prates (1999), a calda sulfocálcica, embora seja um produto de amplo espectro, é inócua a mamíferos e apresenta seletividade moderada a inimigos naturais. Contudo, no presente trabalho, esse composto foi classificado como nocivo a A. brasiliensis, causando alta toxicidade ao ácaro na concentração de $3 \%$.

Hassan et al. (1994) constataram que a calda sulfocálcica a $7 \%$ pode ser altamente tóxica aos ácaros fitoseídeos das espécies Phytoseiulus persimilis Athias-Henriot, Amblyseius pontentillae (Garman) e Typhlodromus pyri (Scheuten).

Os acaricidas abamectin, chlorfenapyr, chlorpyrifos, dimetoato e dinocap afetaram significativamente a população de A. brasiliensis, sendo classificados como moderadamente nocivos (Tabela 2). As mortalidades variaram de 56,8 a 96,9\% para esses produtos. A taxa de maior mortalidade foi a registrada para abamectin ( $\mathrm{Mc}_{\mathrm{c}}=96,9 \%$ ), que foi significativamente mais tóxico às fêmeas de $A$. brasiliensis que os demais acaricidas incluídos na classe 3.

Childers et al. (2001) verificaram que os acaricidas abamectin, chlorfenapyr e dimetoato foram altamente tóxicos ao estigmeídeo $A$. industani, em condições de campo. Resultados semelhantes foram constatados para o ácaro Agistemus fleschneri (Summers), quando exposto ao acaricida dinocap (Nelson et al., 1973).

Os inseticidas thiametoxam e acephate causaram mortalidades de 65,1 e 48,2\% em fêmeas de $A$. brasiliensis, respectivamente, sendo classificados como levemente nocivos (Tabela 2). Acrinathrin, bifenthrin, carbosulfan, deltamethrin, dicofol, flufenoxuron, hexythiazox e spirodiclofen provocaram mortalidades de 1,02 a 15,3\% em A. brasiliensis, significativamente menores que as obtidas para os produtos anteriormente citados (Figura 1).

Carbosulfan foi inócuo à população de $A$. brasiliensis (Tabela 2), resultado semelhante ao observado por Childers et al. (2001), que verificaram que os carbamatos carbaril e formetanate praticamente não afetaram a população de $A$. industani em citros.
Os piretroides acrinathrin, bifenthrin e deltamethrin foram inócuos ao predador $A$. brasiliensis (Tabela 2). Resultados semelhantes, indicando baixa toxicidade aos ácaros estigmeídeos, a esse grupo químico têm sido reportados por vários autores (Villanueva \& Harmsen, 1998; Bostanian \& Laroeque, 2001; Sato et al., 2001).

Embora os piretroides, de modo geral, apresentem baixa toxicidade aos ácaros estigmeídeos, tem sido reportada alta toxicidade a ácaros fitoseídeos em diversas culturas, inclusive em citros (Zacharda \& Hlùchy, 1991; Yamamoto et al., 1992; Sato et al., 1996; Reis et al., 1998; Monteiro, 2001).

Em relação ao efeito na reprodução (Er) de $A$. brasiliensis, constatou-se que o acaricida abamectin não interferiu negativamente na reprodução e viabilidade dos ovos do estigmeídeo $(\mathrm{Er}=1,26)$. Da mesma forma, deltamethrin não afetou a oviposição e a viabilidade dos ovos do predador $(E r=1,0)$ (Tabela 2).

Os valores de Er para os produtos acephate, acrinathrin, bifenthrin, thiametoxam e carbosulfan foram ligeiramente inferiores a $1(0,70 \leq \mathrm{Er} \leq 0,93)$, o que indica pequeno efeito sobre a reprodução e viabilidade dos ovos. Dinocap e chlorpirifos apresentaram efeito ligeiramente mais pronunciado, com Er entre 0,52 e 0,58 (Tabela 2).

Dimetoato e chlorfenapyr proporcionaram efeito bem mais acentuado na reprodução e viabilidade dos ovos do estigmeídeo $A$. brasiliensis com valores de Er de 0,19 e 0,14, respectivamente (Tabela 2).

Dicofol não interferiu na oviposição de $A$. brasiliensis, porém afetou drasticamente a viabilidade dos ovos, apresentando Er de 0,23 (Tabela 2).

Devido à alta mortalidade de fêmeas de $A$. brasiliensis (100\%), causada por cyhexatin, óxido de fenbutatin, propargite, pyridaben e calda sulfocálcica, não foi possível avaliar o efeito desses produtos sobre a reprodução do acarino (Tabela 2).

Com relação aos acaricidas flufenoxuron, hexythiazox e spirodiclofen, apesar de não causarem mortalidade acentuada (Mc $\leq 18,1)$ de $A$. brasiliensis, houve inviabilidade de $100 \%$ dos ovos postos, sendo classificados como nocivos (Tabela 2). Esse fato pode estar relacionado ao mecanismo de ação desses produtos, uma vez que atuam sobre a fecundidade das fêmeas e/ou viabilidade dos ovos (Campos \& Omoto, 2002; Wolf \& Schnorbach, 2002; Rastegari \& Subrahmanyam, 2003).

Reis et al. (2005) avaliaram a mortalidade e a reprodução de fêmeas adultas de ácaros predadores da família Phytoseiidae após o contato residual com 
spirodiclofen e concluíram que o acaricida apresenta algum efeito prejudicial a esses inimigos naturais, incluindo o acaricida na classe 2 para Euseius citrifolius Denmark \& Muma e na classe 3 para Euseius alatus DeLeon.

Dos vinte produtos testados para $A$. brasiliensis, 20\% foram incluídos na classe 1 (inócuo), 10\% na classe 2 (levemente nocivo), 30\% na classe 3 (moderadamente nocivo) e $40 \%$ na classe 4 (nocivo). A maioria dos agrotóxicos testados mostrouse prejudicial ao predador, com $70 \%$ dos produtos classificados como moderadamente nocivos e nocivos.

O agrotóxico carbosulfan foi considerado no presente estudo como inócuo a A. brasiliensis. Este produto é utilizado no controle de ácaros fitófagos, de grande importância na citricultura, tais como Phylocoptruta oleivora (Ashemead) e Polyphagotarsonemus latus (Banks) (Eriophyidae, Tarsonemidae) (Agrofit, 2008). Apesar de carbosulfan mostrar-se inócuo a $A$. brasiliensis, tem sido constatado efeito nocivo desse ingrediente ativo em ácaros fitoseídeos na citricultura (Yamamoto \& Bassanezi, 2003).

Os inseticidas deltamethrin e thiametoxam, classificados como inócuo e levemente nocivo a $A$. brasiliensis, respectivamente, são utilizados para o controle de insetos, como cochonilhas, moscas-dasfrutas, cigarrinhas e outras pragas de grande importância econômica na citricultura (Agrofit, 2008). Thiametoxam tem sido amplamente empregado em citros, para o controle de Diaphorina citri
Kuwayama (Hemiptera: Psyllidae), vetor do "greening”, doença que tem provocado grandes perdas à citricultura brasileira (Fundecitrus, 2008). A baixa toxicidade desses ingredientes ativos sobre $A$. brasiliensis pode ser favorável para o manejo de ácaros-praga, em pomares com presença desse predador.

Segundo Hoy \& Conley (1987), agrotóxicos com baixa toxicidade ( $<50 \%$ de mortalidade) sobre ácaros predadores, em laboratório, também apresentam baixo impacto sobre esses inimigos naturais no campo. Assim sendo, acephate ( $\mathrm{Mc}=$ 48,2\%) poderia ser utilizado em pomares cítricos sem afetar a população de $A$. brasiliensis.

Acrinathrin e bifenthrin foram considerados inócuos (classe 1) a A. brasiliensis. Estes acaricidas, segundo AGROFIT, (2008), são recomendados para o controle de $B$. phoenicis e podem ser utilizados em programa de MIP em citros, sem afetar a população de $A$. brasiliensis no campo. A manutenção desse estigmeídeo nos pomares cítricos pode propiciar maior eficiência de controle das populações dos ácaros fitófagos, minimizando as aplicações de acaricidas e reduzindo o custo final da produção.

Ressalta-se que os resultados obtidos neste trabalho, complementados com trabalhos de campo, poderão contribuir para uma escolha mais adequada dos agrotóxicos a serem utilizados em programas de manejo integrado, em pomares de citros, onde $A$. brasiliensis esteja presente.

TABELA 1 - Relação dos tratamentos estabelecidos para o bioensaio de seletividade de agrotóxicos em Agistemus brasiliensis. Campinas-SP, 2008.

\begin{tabular}{|c|c|c|c|c|}
\hline Produto técnico & $\begin{array}{l}\text { Produto Comercial / } \\
\text { Formulação }\end{array}$ & $\mathrm{Uso}^{1}$ & $\begin{array}{c}\text { Dosagem (produto } \\
\text { comercial em 100L) }\end{array}$ & $\begin{array}{c}\text { Classe }^{2} \\
\text { toxicológica }\end{array}$ \\
\hline abamectin & Vertimec $18 \mathrm{CE}$ & A & $30 \mathrm{~mL}$ & III \\
\hline acephate & Orthene $750 \mathrm{PM}$ & A, I & $100 \mathrm{~g}$ & III \\
\hline acrinathrin & Rufast $50 \mathrm{SC}$ & A & $10 \mathrm{~mL}$ & IV \\
\hline bifenthrin & Talstar $100 \mathrm{CE}$ & A & $20 \mathrm{~mL}$ & II \\
\hline calda sulfocálcica & - & $\mathrm{A}, \mathrm{I}, \mathrm{F}$ & $3.000 \mathrm{~mL}$ & - \\
\hline carbosulfan & Marshal 200 SC & $\mathrm{A}, \mathrm{I}$ & $50 \mathrm{~mL}$ & II \\
\hline cyhexatin & Sipcatin $500 \mathrm{SC}$ & A & $50 \mathrm{~mL}$ & III \\
\hline chlorfenapyr & Citrex $240 \mathrm{SC}$ & A, I & $62,5 \mathrm{~mL}$ & II \\
\hline chlorpyrifos & Lorsban $480 \mathrm{CE}$ & A, I & $200 \mathrm{~mL}$ & II \\
\hline deltamethrin & Decis $25 \mathrm{CE}$ & I & $50 \mathrm{~mL}$ & II \\
\hline dicofol & Kelthane $480 \mathrm{CE}$ & A & $77 \mathrm{~mL}$ & II \\
\hline dimetoato & Perfekthion $400 \mathrm{CE}$ & A, I & $190 \mathrm{~mL}$ & $\mathrm{I}$ \\
\hline dinocap & Karathane $369 \mathrm{CE}$ & A & $50 \mathrm{~mL}$ & II \\
\hline flufenoxuron & Cascade $100 \mathrm{SC}$ & A & $30 \mathrm{~mL}$ & I \\
\hline hexythiazox & Savey PM & A & $3 \mathrm{~g}$ & III \\
\hline óxido de fenbutatin & Torque $500 \mathrm{SC}$ & A & $80 \mathrm{~mL}$ & III \\
\hline propargite & Omite $720 \mathrm{CE}$ & A & $100 \mathrm{~mL}$ & II \\
\hline pyridaben & Sanmite $200 \mathrm{CE}$ & A & $75 \mathrm{~mL}$ & I \\
\hline spirodiclofen & Envidor $240 \mathrm{SC}$ & A & $20 \mathrm{ml}$ & III \\
\hline thiametoxam & Actara $250 \mathrm{WG}$ & $\mathrm{I}$ & $20 \mathrm{~g}$ & III \\
\hline
\end{tabular}

${ }^{1} \mathrm{~A}=$ acaricida; $\mathrm{I}$ = inseticida; $\mathrm{F}$ = fungicida

${ }^{2} \mathrm{I}$ = extremamente tóxico, $\mathrm{II}$ = altamente tóxico; III = medianamente tóxico, IV = pouco tóxico 
TABELA 2 - Seletividade de agrotóxicos sobre fêmeas adultas de Agistemus brasiliensis em folhas de citros. Campinas-SP, 2008.

\begin{tabular}{|c|c|c|c|c|c|c|}
\hline Produto técnico & $\begin{array}{l}\text { Concentração } \\
\text { g de i.a. } / \mathbf{1 0 0 L}\end{array}$ & $\begin{array}{c}\text { Mc }^{1} \text { e erro- } \\
\text { padrão da } \\
\text { média }\end{array}$ & $\begin{array}{c}\text { Sobreviventes } \\
100 \%-M_{c}\end{array}$ & $\mathbf{E}_{\mathbf{r}}^{2}$ & $\mathbf{E}^{3}$ & Classe $^{4}$ \\
\hline abamectin & 0,54 & $96,90 \pm 1,02$ & 3,10 & 1,26 & 96,10 & 3 \\
\hline acephate & 75,00 & $48,20 \pm 3,61$ & 51,80 & 0,70 & 63,74 & 2 \\
\hline acrinathrin & 0,50 & $1,02 \pm 0,00$ & 98,98 & 0,93 & 7,95 & 1 \\
\hline bifenthrin & 2,00 & $13,84 \pm 3,20$ & 86,16 & 0,90 & 22,46 & 1 \\
\hline calda sufocálcica & $3.000 *$ & 100,00 & 0,00 & - & 100,00 & 4 \\
\hline carbosulfan & 10,00 & $2,20 \pm 2,54$ & 87,80 & 0,79 & 22,74 & 1 \\
\hline cyhexatin & 25,00 & 100,00 & 0,00 & - & 100,00 & 4 \\
\hline chlorfenapyr & 15,00 & $59,00 \pm 4,72$ & 41,00 & 0,14 & 94,26 & 3 \\
\hline chlorpyrifos & 96,00 & $65,60 \pm 9,71$ & 34,40 & 0,58 & 80,05 & 3 \\
\hline deltamethrin & 1,25 & $2,00 \pm 1,07$ & 98,00 & 1,00 & 2,00 & 1 \\
\hline dicofol & 37,00 & $15,32 \pm 3,20$ & 84,68 & 0,23 & 80,53 & 3 \\
\hline dimetoato & 76,00 & $56,80 \pm 9,20$ & 43,20 & 0,19 & 91,79 & 3 \\
\hline dinocap & 18,45 & $75,10 \pm 6,09$ & 24,90 & 0,52 & 87,05 & 3 \\
\hline flufenoxuron & 3,00 & $7,10 \pm 1,77$ & 92,90 & 0,00 & 100,00 & 4 \\
\hline hexythiazox & 1,50 & $5,00 \pm 1,02$ & 95,00 & 0,00 & 100,00 & 4 \\
\hline óxido de fenbutatin & 40,00 & 100,00 & 0,00 & - & 100,00 & 4 \\
\hline propargite & 72,00 & 100,00 & 0,00 & - & 100,00 & 4 \\
\hline pyridaben & 15,00 & 100,00 & 0,00 & - & 100,00 & 4 \\
\hline spirodiclofen & 4,80 & $18,10 \pm 2,48$ & 81,90 & 0,00 & 100,00 & 4 \\
\hline thiametoxam & 5,00 & $65,10 \pm 4,54$ & 34,90 & 0,93 & 67,55 & 2 \\
\hline
\end{tabular}

${ }^{1}$ Mortalidade corrigida: $\mathrm{M}_{c}$; ${ }^{2}$ Efeito na reprodução: Er= R trat. / R test.; ${ }^{3}$ Efeito total: E\%=100\% -(100\%-Mc) x Er; ${ }^{4} \mathrm{Classes}$ de toxicidade segundo IOBC. - *Concentração do produto formulado (mL/100 L).

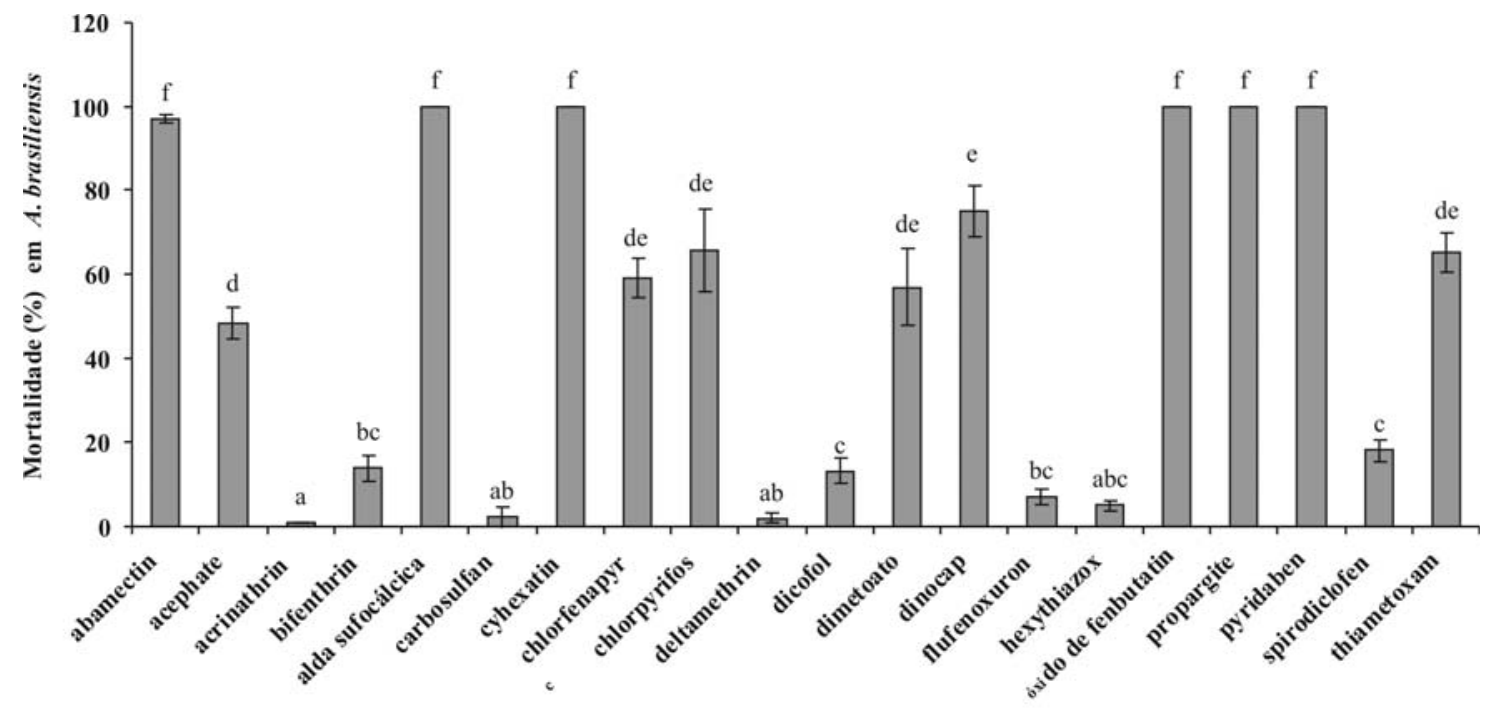

FIGURA 1 - Mortalidade corrigida e ( \pm EP) de fêmeas de Agistemus brasiliensis Matioli, Ueckermann \& Oliveira, avaliada 72 horas após a aplicação dos produtos. Colunas com mesma letra não diferem estatisticamente entre si, ao nível de 5\%, pelo teste Tukey. 


\section{CONCLUSÕES}

1-Os produtos acrinathrin, bifenthrin, carbosulfan e deltamethrin mostram-se inócuos (classe 1) ao estigmeídeo A. brasiliensis.

2- Acephate e thiametoxam (classe 2) são levemente nocivos a $A$. brasiliensis.

3-Calda sulfocálcica, cyhexatin, flufenoxuron, hexythiazox, óxido de fenbutatin, propargite, pyridaben e spirodiclofen (classe 4) mostram-se nocivos a A. brasiliensis.

\section{REFERÊNCIAS}

AGROFIT. Sistema de Agrotóxicos Fitossanitários, do Ministério da Agricultura, Pecuária e Abastecimento. Disponível em: <http:extranet.agricultura.gov.br/agrofit_cons/ principal_agrofit_cons>. Acesso em: 20 fev. 2008.

BAKKER, F.M.; GROVE, A.; BLUMEL, S.; CALIS, J.; OOMEN, P. Side-effect test for phytoseiids and their rearing methods. IOBC/WPRS Bulletin, Montfavest, v.15, n.3, p.61-81, 1992.

BOSTANIAN, N.J.; LAROEQUE, N. Laboratory tests to determine the intrinsic toxicity of four fungicides and two insecticides to the predacious mite Agistemus fleschneri. Phytoparasitica, Jerusalém, v.29, n.3, p.1-8, 2001.

CAMPOS, F.J.; OMOTO, C. Resistance to hexythiazox in Brevipalpus phoenicis (Acari: Tenuipalpidae) from Brazilian citrus. Experimental and Applied Acarology, Amsterdam, v.26, p.243-251, 2002.

CHILDERS, C.C.; VILLANUEVA, R.; AGUILAR, H.; CHEWNING, R.; MICHAUD, J.P. Comparative residual toxicities of pesticides to the predator Agistemus industani (Acari: Stigmaeidae) on citrus in Florida. Experimental and Applied of Acarology, Amsterdam, v. 25, p. 461-474, 2001.

FUNDECITRUS. Ajuda no controle do Greening. Fundo de Defesa da Citricultura. Disponível em <http:/ www.fundecitrus.com.br/doencas/greening.html> Acesso em 10 out. 2008.

GERSON,U.; SMILEY, R.L.; OCHOA, R. Mites(Acari) for pest control.Oxford: Blackell Science Ltda, 2003, 539p.
HASSAN, S.A.; BIGLE, F.; BLAISINGER, P.; BOGENSHUTZ, H.; BRUN, J.; CHIVERTON, P.; DICKLER, E.;EASTERBROOK, M.A.;EDWARDS,P.J.; ENGLERT, W.D.; FIRTH, S.I.; HUANG, P.; INGLESFIELD, D.; KLINGAUF, F.; KUHNER, C.; OVERMEER, W.P.J.; PLEVOETS, P.; REBOULET, J.N.; RIECKMANN,W.; SAMSOE-PETERSEN, L.; SHIRES, S.W.; STAUBLI, A.; STEVENSON, J.; TUSET, J.J.; VANWETSWINKEL, G.; VANZON, Q. Standard method to test the side-effects of pesticides on natural enemies of insects and mites developed by the IOBC/ WPRS - working group "Pesticides and Beneficial Organisms”. EPPO Bulletin, Oxford, v.15, p.214-255, 1985.

HASSAN, S.A.; BIGLE, F.; BOGENSCHUTZ, H.; BOLLER, E.; BRUN, J.; CHIVERTON, P.; EDWARDS, P.J.; MANSOUR, F.; NATON, E.; OOMEN, P.A.; OVERMEER, W.P.J.; POLGAR, L.; RIECKMANN, W.; SAMSOE-PETERSEN, L.; STAUBLI,A.; STERK, G.; TAVARES, K.; TUSET, J.J.; VIGGIANI, G.; VIVAS, A.G. Result of the sixth joint pesticide testing programme of the IOBC/WPRS - working group "Pesticides and Beneficial Organisms". Entomophaga, Paris, v.39, n.1, p.107-119, 1994.

HENDERSON, C.F.; TILTON, E.W. Tests with acaricides against the brown wheat mite. Journal Economic Entomology, Lanham, v.63, p.1536-1539, 1955.

HOY, M.A.; CONLEY, J. Toxicity of pesticides to western predatory mite. California Agriculture, Oakland, v.41, n.7/8, p.12-14, 1987.

KITAJIMA, E.W.; MULLER, G.M.; COSTA, A.S. Partículas basiliformes associadas à leprose dos citros. In:CONGRESSOBRASILEIRODE FRUTICULTURA, 1., 1971. Campinas. Anais, 1971. p.419-438.

KOMATSU, S.S. Aspectos bioecológicos de Euseius concordis (Chant, 1959) (Acari: Phytoseiidae) e seletividade dos acaricidas convencionais nos citros. 1988. 117f. Dissertação (Mestrado em Agronomia) Escola Superior de Agricultura "Luiz de Queiroz", Piracicaba, SP.

KRANTZ, G.W. Amanual of acarology. 2.ed. Corvalis: Oregon St. Univ. Book Stores, 1978. 509 p.

MATIOLI, A.L. Aspectos taxonômicos e bioecológicos de ácaros predadores Stigmaeidae (Acari) de ocorrência em citros. 2002. 82f. Tese (Doutorado em Agronomia, Área de Concentração em Entomologia Agrícola) - Faculdade de Ciências 
Agrárias e Veterinárias, Universidade Estadual Paulista, Jaboticabal-SP.

MATIOLI, A.L.; OLIVEIRA, C.A.L. de. Biologia de Agistemus brasiliensis Matioli, Ueckermann \& Oliveira (Acari: Stigmaeidae) e sua potencialidade de predação sobre Brevipalpus phoenicis (Geijskes) (Acari: Tenuipalpidae). Neotropical Entomology, Vacaria, v.36, n.4, p.557-582, 2007.

McMURTRY, J. A.; HUFFAKER, C. B.; VAN DEVRIE, M. Ecology of Tetranychidae mites and their natural enemies: A review. I. Tetranychidae enemies: Their biological characters and the impact of spray practices. Hilgardia, Berkeley, v. 40, n. 11, p. 331390, 1970.

MINEIRO, L.C. de; SATO, M.E.; RAGA, A. Population dynamics of phytophagous and predaceous mite on coffee in Brazil, with emphasis on Brevipalpus phoenicis (Acari: Tenuipalpidae). Experimental and Applied Acarology, Amsterdam, v.44, p.277-291, 2008.

MONTEIRO, L.B. Seletividade de inseticidas a Neoseiulus californicus McGregor (Acari: Phytoseiidae) em macieira, no Rio Grande do Sul. Revista Brasileira de Fruticultura, Jaboticabal, v.23, n.3 p., 2001.

MORAES, G.J. de. Controle biológico de ácaros fitófagos. Informe Agropecuário. Belo Horizonte, v. 15, n. 167, p. 56-62, 1991.

MORAES, G. J. de. Controle biológico de ácaros fitófagos com ácaros predadores. p.225-237. In: J. R. Parra, P. S. M. Botelho, B. S. Corrêa-Ferreira \& J.M.S. Bento (Eds). Controle biológico no Brasil -parasitóides e predadores, Barueri, 2002, 609p.

MORAES, G.J. de; SÁ, L.A.N. Perspectivas do controle biológico do ácaro da leprose em citros In: OLIVEIRA, C.A.L. de; DONADIO L.C. (Ed.). Leprose dos citros. Jaboticabal: FUNEP, 1995. p.117-128.

MOREIRA, P.H.R. Ocorrência, dinâmica populacional de ácaros predadores em citros e biologia de Euseius citrifolius (Acari: Phytoseiidae). 1993. 110f. Dissertação (Mestrado em Agronomia) Faculdade de Ciências Agrárias e Veterinárias, Universidade Estadual Paulista, Jaboticabal-SP.
NELSON, E.E.; CROFT, B.A.; HOWITT,A.J.; JONES, A.L. Toxicity of apple orchard pesticides to Agistemus fleschneri. Environmental Entomology, Lanham, v.2, p.219-222, 1973.

OMOTO, C.; ALVES, E.B.; RIBEIRO, P.C. Detecção e monitoramento da resistência de Brevipalpus phoenicis (Geijskes) (Acari: Tenuipalpidae) ao dicofol. Anais da Sociedade Entomológica do Brasil, Itabuna, v.29, n.4, p.757-764, 2000.

OVERMEER, W.P.J. Laboratory method for testing sideeffects of pesticides on the predaceous mite Typhlodromalus pyri and Amblyseius potentillae (Acari: Phytoseiidae). IOBC/WPRS Bulletin, Montfovet, v.11, n.4, p. 65-69, 1988.

PRATES, H.S. Calda bordalesa, sulfocálcica e viçosa - produtos alternativos na citricultura. CATI Responde, n.40, 1999. Disponível em: <http:// www.cati.sp.gov.br>. Acesso em: 21 jun. 2003.

RASMY, A.H. Mass rearing of the predator mite Agistemus exsertus. Anzeiger-Fur-Schadlingskunde,Pflanzen-Und-Umweltschutz, Berlin, v.48, p.55-56, 1975.

RASTEGARI, N.; SUBRAHMANYAM, B. Sublethal effects of flufenoxuron, fipronil and methoxyfenozide on Spodoptera litura (Fabricius). Pesticide Research Journal, New Delhi, v.15, n.2, p.168-172, 2003

REIS, P.R.; CHIAVEGATO, L.G.; MORAES, G.J. de; ALVES, E.B.; SOUSA, E.O. Seletividade de agroquímicos ao ácaro predador Iphiseiodes zuluagai Denmark \& Muma (Acari: Phytoseiidae). Anais da Sociedade Entomológica do Brasil, Itabuna, v.27, n.2, p.265-274. 1998.

REIS, P.R.; SOUSA, E.O.; ALVES, E.B. Seletividade de produtos fitossanitários ao ácaro predador Euseius alatus De Leon( Acari: Phytoseiidae). Revista Brasileira de Fruticultura, Jaboticabal, v.21, n.3, p.350355, 1999.

REIS, P.R.; PEDRO NETO, M.; FRANCO, R.A. Controle de Brevipalpus phoenicis (Geijskes, 1939) e Oligonychus ilicis (McGregor, 1917) (Acari: Tenuipalpidae, Tetranychidae) em cafeeiro e o impacto sobre ácaros benéficos. II - Spirodiclofen e Azocyclotin. Ciência e Agrotecnologia, Lavras, v. 29, n. 3, p. 528537, 2005. 
RODRIGUES, J.C.V.; KITAJIMA, E.W.; CHILDERS, C.C.; CHAGAS, C.M. Citrus leprosis virus vectored by Brevipalpus phoenicis (Acari: Tenuipalpidae) in citrus in Brazil. Experimental and Applied Acarology, Amsterdam, v.30, p.161-179, 2003.

SANTOS, M.A.; LAING, J.E. Stigmaeid predators. In: HELLE, W.; SABELIS, M.W. Spider mites. Amsterdam, The Netherlands: Elsevier Science Publishers, 1985. p.197-203.

SATO, M.E. Perspectivas do uso de ácaros predadores no controle biológico de ácaros-praga na citricultura. Laranja, Cordeirópolis, v.26, n.2, p.291-305, 2005.

SATO, M.E.; RAGA, A.; CERÁVOLO, L.C.; ROSSI, A.C.; POTENZA, M.R. Ácaros predadores em pomar cítrico de Presidente Prudente, Estado de São Paulo. Anais da Sociedade Entomológica do Brasil, Itabuna, v. 23, n.3, p.435-441, 1994.

SATO, M.E.; RAGA,A.; CERÁVOLO, L.C.; CEZÁRIO, A.C; ROSSI, A. Efeito da utilização de acaricidas em citros, sobre a população de Brevipalpus phoenicis (Geijskes, 1939) (Acari: Tenuipalpidae) e ácaros predadores (família Phytoseiidae). Scientia Agricola, Piracicaba, v.52, n.2, p.282-286, 1995.

SATO, M.E.; RAGA,A.;CERÁVOLO,L.C.;ROSSI,A.C.; SOUZA FILHO, M.F. de. Toxicidade residual de acaricidas a Iphiseiodes zuluagai Denmark \& Muma, 1972 (Acari: Phytoseiidae). Arquivos do Instituto Biológico, São Paulo, v.63, p.15-19, 1996.

SATO, M.E.; RAGA, A.; CERÁVOLO, L.C.; SOUZA FILHO, M.F.; ROSSI, A.C.; MORAES, G. J. de. Effect of insecticides and fungicides on the interaction between members of mite families Phytoseiidae and Stigmaeidae on citrus. Experimental and Applied Acarology, Amsterdam, v.25, p.809-818, 2001.

SEPASGOSARIAN, H. The world species of the superfamily Raphignatoidea. Zeitschrift fuer Angewandte Zoologia, Frankfurt, v.72, p.437-478, 1985.

SILVA, M.Z. da; OLIVEIRA, C.A.L. de. Seletividade de alguns Agrotóxicos em uso na citricultura ao ácaro predador Neoseiulus californicus (McGregor) (Acari: Phytoseiidae). Revista Brasileira de Fruticultura, Jaboticabal, v.28, n.2, p.205-208, 2006.
SILVA, M.Z. da; OLIVEIRA, C.A.L. de. Toxicidade de alguns agrotóxicos recomendados na citricultura sobre Neoseiulus californicus (McGregor) (Acari: Phytoseiidae). Revista Brasileira de Fruticultura, Jaboticabal, v.29, n.1, p.085-090, 2007.

VAN DE VRIE, M.; McMURTRY, J.A.; HUFFAKER, C.B. Ecology of tetranychid mites and their natural enemies: A review. III. Biology, ecology, and pest status, and host-plant relations of tetranychids. Hilgardia, Berkeley, v.41, p.387-403, 1972.

VILLANUEVA, R.T.; HARMSEN, R. Studies on the role of the stigmaeid predator Zetzellia mali in the acarine system of apple foliage. Proceedings of the Entomological Society of Ontario, Ontario, v.129, p.149-155, 1998.

WOLF, C.; SCHNORBACH, H.J. Ecobiological profile of the acaricide spirodiclofen. PflanzenschutzNachrichten Bayer, Frankfurt, v.55, n.2-3, p.177-187, 2002.

WOOLHOUSE, M. E. J.; HARMSEN, R. The mite complex of the foliage of a pesticide-free apple orchard: population dynamics and habitat associations. Proccedings of Entomology Society, v. 115, p. 1-11, 1994.

YAMAMOTO, P.T.; PINTO, A.S.; PAIVA, P.E.B.; GRAVENA, S. Seletividade de agrotóxicos aos inimigos naturais de pragas dos citros. Laranja, Cordeirópolis, v.13, p.709-755, 1992.

YAMAMOTO, P.T.; BASSANEZI, R.B. Seletividade de produtos fitossanitários aos inimigos naturais de pragas dos citros. Laranja, Cordeirópolis, v.24, n.2, p.353-382, 2003.

ZACHARDA, M.; HLÙCHY, M. Long-term residual efficacy of commercial formulations of pesticides to Typhlodromus pyri Scheuten (Acari: Phytoseiidae) inhabiting commercial vineyards. Experimental and Applied Acarology, Amsterdam, v.13, p.27-40, 1991.

ZAHER, M.A.; ELBADRY, E.A. Life history of the predatory mite Agistemus fleschneri Summers, and effect of nutrition on its biology. Bulletin Entomological of Society Egypt, Cairo, v. 45, p. 375-385, 1961. 